\title{
An analytical approach to transient homovalent cation exchange problems
}

\author{
Xavier Sanchez-Vila, Diogo Bolster* \\ Dept. of Geotechnical Engineering and Geosciences, Technical University of Catalonia, Jordi Girona 31, 08034 Barcelona, Spain
}

\section{A R T I C L E I N F O}

\section{Article history:}

Received 2 April 2009

Received in revised form 20 July 2009

Accepted 21 September 2009

This manuscript was handled by L. Charlet Editor-in-Chief, with the assistance of Carlos Ayora, Associate Editor

\section{Keywords:}

Cation exchange

Groundwater

Analytical

\begin{abstract}
S U M M A R Y
Cation exchange in groundwater is one of the dominant surface reactions that occurs in nature and it carries with it many important environmental implications. The mass transfer of cation exchanging pollutants in groundwater can be described by a series of coupled partial differential equations, involving both aqueous and adsorbed species. The resulting system is mathematically challenging due to the complex nonlinearities that arise, which in turn complicates analytical approaches. While some analytical solutions for simplified problems exist, these typically lack the mechanisms that allow the waters to change their global chemical signature (in terms of total cations present in aqueous form) over time. We propose a methodology to solve the problem of exchanging two homovalent cations by deriving the driving equation for one of the aqueous species. This equation incorporates explicitly a retardation factor and a decay term, both with parameters that can vary in space and time. While the full solution can only be obtained numerically, we provide a solution in terms of a perturbative approach, where the leading terms can be obtained explicitly. The resulting solution provides physical explanations for the possible existence of non-monotonic concentrations for a range of parameters governing cation exchange processes.
\end{abstract}

(ㄷ) 2009 Elsevier B.V. All rights reserved.

\section{Introduction}

Many natural and artificial substances are characterized as being electrically unbalanced. Thus, free charges (often negative) are surrounded by cations in order to achieve neutrality. The links formed between cations and minerals are relatively weak such that if other cations exist the process of exchange can take place. The result is a surface reaction. Cation binding and exchange plays a fundamental role in a number of environmental and industrial applications, including bacterial growth (Maurya and Subramaniam, 2007; Shephard et al., 2008), refinement of fuel products (Bhattacharyya et al., 2006), development of superconducting materials (Xing et al., 2006), and elaboration of food products (protein separation), among others. The cation-exchange capacity of different natural and artificial materials can be used to remove undesired molecules from water, such as dyes (Wu et al., 2008), enzymes (Turmanova et al., 2005), etc. Applications of cation exchange to daily life problems include household water softeners, where hard water flows through a resin that exchanges ions $\mathrm{Ca}^{2+}$ and $\mathrm{Mg}^{2+}$ with $\mathrm{Na}^{+}$.

Cation exchange processes occur naturally in groundwater systems. Due to the presence of clay minerals aquifers can often be regarded as weak cation exchangers. These minerals can act as sorbants to major cations, which can then be exchanged with other

\footnotetext{
* Corresponding author. Tel.: +34 655039065.

E-mail address: diogobolster@gmail.com (D. Bolster).
}

cations when water with a given chemical signature reaches the aquifer. This has important implications in solute transport applications, including freshening of marine sediments (Walraevens et al., 2007; Lambrakis, 2006), landfill leaching (Bjerg and Christensen, 1993), industrial pollution problems (Appelo et al., 1993), saltwater intrusion problems (Carlyle et al., 2004; Bolster et al., 2007), or artificial recharge and recovery (Bouwer, 2002; McNab et al., 2009).

A given aquifer may contain materials which have a strong natural tendency to exchange one ion for another. Naturally occurring exchangers display different cation selectivities (Appelo, 1994b). As a consequence, different cations have different retardation coefficients, an effect that is used for chromatographic separation of compounds in a given mixture (Cernik et al., 1994; Appelo, 1994a). When a number of cations are present in the solution, transport of those cations is frequently observed as a series of retarded fronts, each one clearly separable from the others (Timms and Hendry, 2007). The conditions necessary for such fronts to develop are analyzed in Appelo et al. (1993), among others. In practice, the values of actual retardation coefficients can be quite large (e.g., Bjerg and Christensen (1993) observed fronts that traveled 20 times slower than the flow speed).

To date, the governing equations for cation transport are usually written as an extension of the advection-dispersion equation with additional sink/source terms to account for the ion exchange processes. This is then combined with a one-site cation-exchange equation, which is derived independently from batch tests. While this model often reproduces observed breakthrough curves (e.g. 
Schulin et al., 1989), it is also true that a better reproduction of observed data can be obtained by using a multiple-site model (Vulava et al., 2000, 2002). This is the case for certain cations such as Cs, where one-site models never appear to faithfully reproduce data (Steefel et al., 2003). One of the drawbacks of multi-site models is that the resulting distributions of cation-exchange selectivities are strictly empirical (Vulava et al., 2000).

In any case, the equations governing cation exchange in groundwater are highly non-linear. Thus, solutions are usually sought by numerical means, and a wide range of codes that include multication exchange processes are available. Conversely, a limited number of published analytical solutions exist for such a complex problem (and always introducing some restrictive simplifications). An initial analytical solution was obtained for the purely advective case (Harmsen and Bolt, 1982). Combined analytical-numerical solutions exist and are based on the fact that all governing equations can be combined in terms of a single highly non-linear partial differential equation (Valocchi et al., 1981; Lewis et al., 1987). Following Valocchi's approach (Valocchi et al., 1981), Dou and Jin (1996) used the method of characteristics with a special treatment of shock waves to derive a closed-form solution for binary homovalent ion exchange and transport in a 1D infinite domain. Jin and Ye, 1999 used a similar approach to derive an approximate analytical solution for monovalent-divalent ion exchange transport. In these last two works it is assumed that the displacing and the displaced water have different chemical composition in terms of aqueous concentrations of the two cations, but that the sum of the two concentrations must be €qual. While the solytions provided in Dou and Jin (1996) and and useful, they lack the capacity to capt ing water has a different total sum of unrealistic in practice. Thus in most sought numerically, in particular when changes in chemical composition

In this work we use the component method to rederive the nonlinear governing equation for a homovalent problem where total

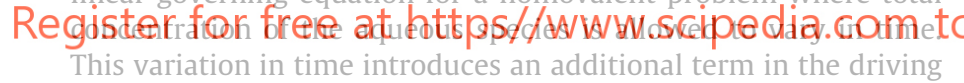
equation with respect to existing approaches, which renders the system highly non-linear. In spite of that, under some simplifying assumptions we present an approximate analytical solution that can be obtained by means of a perturbation approach, which illustrates potential mass accumulation/decrease at the edge of a moving wave.

\section{Binary problem formulation}

Defining the problem in terms of a coupled system of equations

The problem we consider looks at the evolution of the concentration of two cations in aqueous or adsorbed phases that can be exchanged. We consider a groundwater system initially in chemical equilibrium between the rock matrix and the resident water. The system is then invaded by another water containing a different concentration of the exchanging cations. Thus, cation exchange takes place locally to reequilibrate the system with the mixture of existing and invading waters. Additionally, the invading water can change its composition with time, to account for water coming from a landfill or an industrial site, so that a final constant concentration might not be reached at any point in space. The dominant transport processes considered in this work are advection, dispersion and cation exchange, but additional processes could be included in the formulation.

The problem may be expressed as a set of coupled equations satisfied by the four dependent variables (two aqueous concentra- tions and two adsorbed ones). The first two equations account for mass balance of each dissolved cation.

$$
\begin{aligned}
& \frac{\partial c_{1}}{\partial t}+\frac{\gamma}{z_{1}} \frac{\partial \beta_{1}}{\partial t}=-\mathbf{v} \nabla c_{1}+\nabla\left(\mathbf{D} \nabla c_{1}\right) \\
& \frac{\partial c_{2}}{\partial t}+\frac{\gamma}{z_{2}} \frac{\partial \beta_{2}}{\partial t}=-\mathbf{v} \nabla c_{2}+\nabla\left(\mathbf{D} \nabla c_{2}\right)
\end{aligned}
$$

where $c_{\mathbf{j}}(\mathbf{j}=1,2)$ is the aqueous concentration of the $\mathbf{j}$ th dissolved cation $\left[\mathrm{mmol} \mathrm{L}^{-3}\right] ; \beta_{\mathbf{j}}$ the (normalized) equivalent fraction of the $\mathbf{j}$ th exchange cation $[-] ; z_{\mathbf{j}}$ the corresponding valence; $\mathbf{v}$ water velocity; and $\mathbf{D}$ is the dispersion tensor. The second term on the left hand side of (1) and (2) accounts for the exchange between the aqueous and the adsorbed phase. The constant $\gamma$ is calculated from the cation-exchange capacity (CEC) $[\mathrm{mmol} / \mathrm{M}]$, bulk density $\left(\rho_{b}\left[M L^{-3}\right]\right)$, and porosity $(\phi[-])$, as

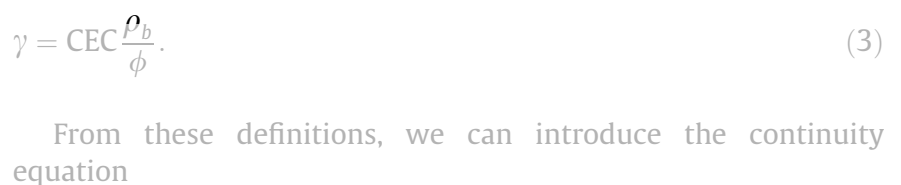
equation

\section{$\beta_{1}+\beta_{2}=1$}

In order to close the system we need a fourth equation relating the aqueous and adsorbed concentrations. Here we adopt the Gaines-1homas nodel (Gaines and Thoinas, 1953). Though con-
tested in some works (e.g., Garlyle et al., 2004), the Gaines-Thomas
convention is perhaps the most widely used in practical applica-
tions. Vulava et al. (2000) compare different cation exchange inod-
els and find that the Gaines-Thomas provides the best fit to
experimental data. The Gaines-Thomas equilibrium/equation is gi-
ven by

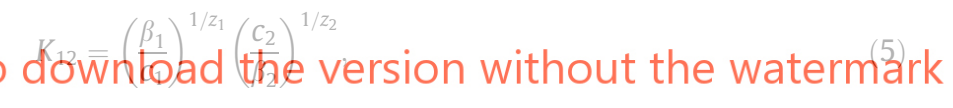
where $K_{12}$ is an experimentally determined constant.

Decoupling the system of equations for the homovalent problem

The system of Eqs. (1)-(5) can be rewritten as a sequence of decoupled equations. We start by defining a new quantity which corresponds to the total amount of positive charges of exchangeable cations present in aqueous form,

$\mathrm{u}=\sum_{j=1}^{2} z_{j} c_{j}$

Table 1

Parameters considered for the sample case.

\begin{tabular}{ll}
\hline Parameter & Value \\
\hline & \\
CEC & 0.2 \\
$\rho_{b}$ & $1 \times 10^{-2} \mathrm{mmol} / \mathrm{g}$ \\
$\phi$ & $1.8 \mathrm{~g} / \mathrm{cm}^{3}$ \\
$\gamma$ & 0.2 \\
$\bar{u}$ & $90 \mathrm{mmol} / \mathrm{L}$ \\
$C_{1 i}$ & $10 \mathrm{mmol} / \mathrm{L}$ \\
$C_{1 f}$ & 0.02 \\
$C_{2 i}$ & 0.01 \\
$C_{2 f}$ & 0.73 \\
$z_{1}$ & 1.24 \\
\hline
\end{tabular}


Combining (1), (2), and (4), this quantity (which in general varies in space and time) is seen to be driven by a conservative ADE equation

$\frac{\partial u}{\partial t}=-\mathbf{v} \nabla u+\nabla(\mathbf{D} \nabla u)$

Using (5), (6) and (4) it is possible to write an explicit expression for $\beta_{1}$ in terms of $c_{1}$ and $u$. The actual expression, denoted heterovalent isotherm, depends on the values of $z_{\mathbf{j}}$ (see e.g. Valocchi et al. (1981) and Jin and Ye (1999) for the monovalent-divalent case). In the particular case of $z_{1}=z_{2}, u$ is most conveniently defined as $u=c_{1}+c_{2}$. Further defining $K=K_{12}^{z_{1}}$ the homovalent isotherm becomes

$\beta_{1}=\frac{K c_{1}}{u-c_{1}+K c_{1}}$

From (8) it follows that

$\frac{\partial \beta_{1}}{\partial t}=\frac{\partial \beta_{1}}{\partial c_{1}} \frac{\partial c_{1}}{\partial t}+\frac{\partial \beta_{1}}{\partial u} \frac{\partial u}{\partial t}$

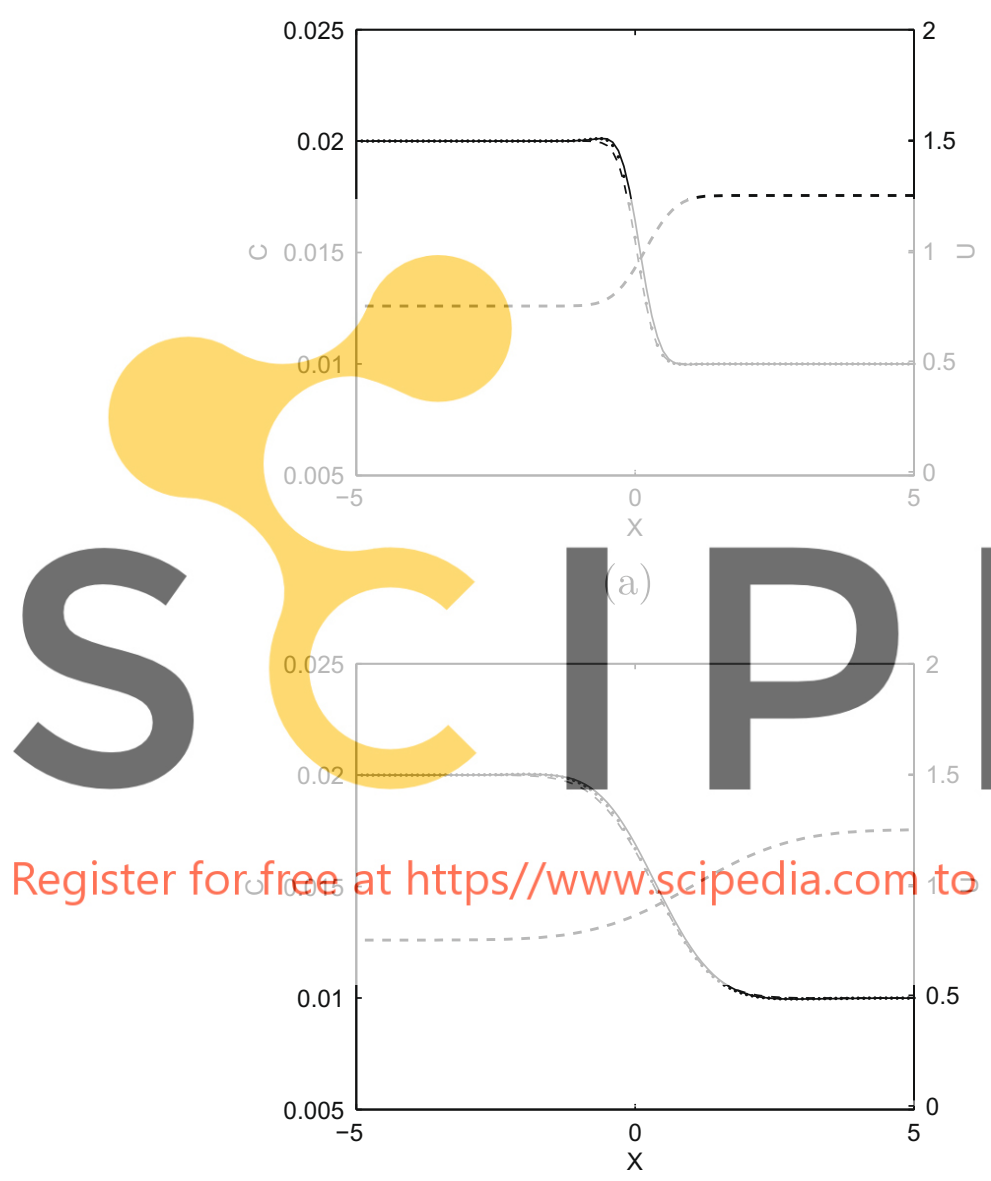

(c)

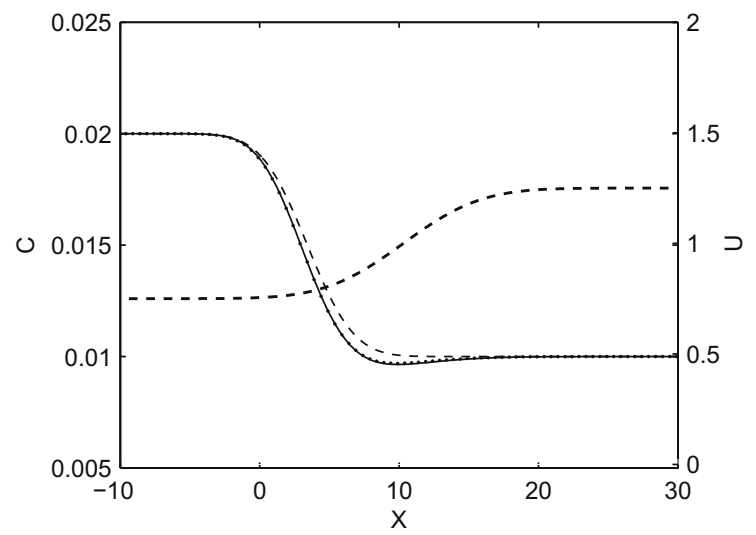

(e)
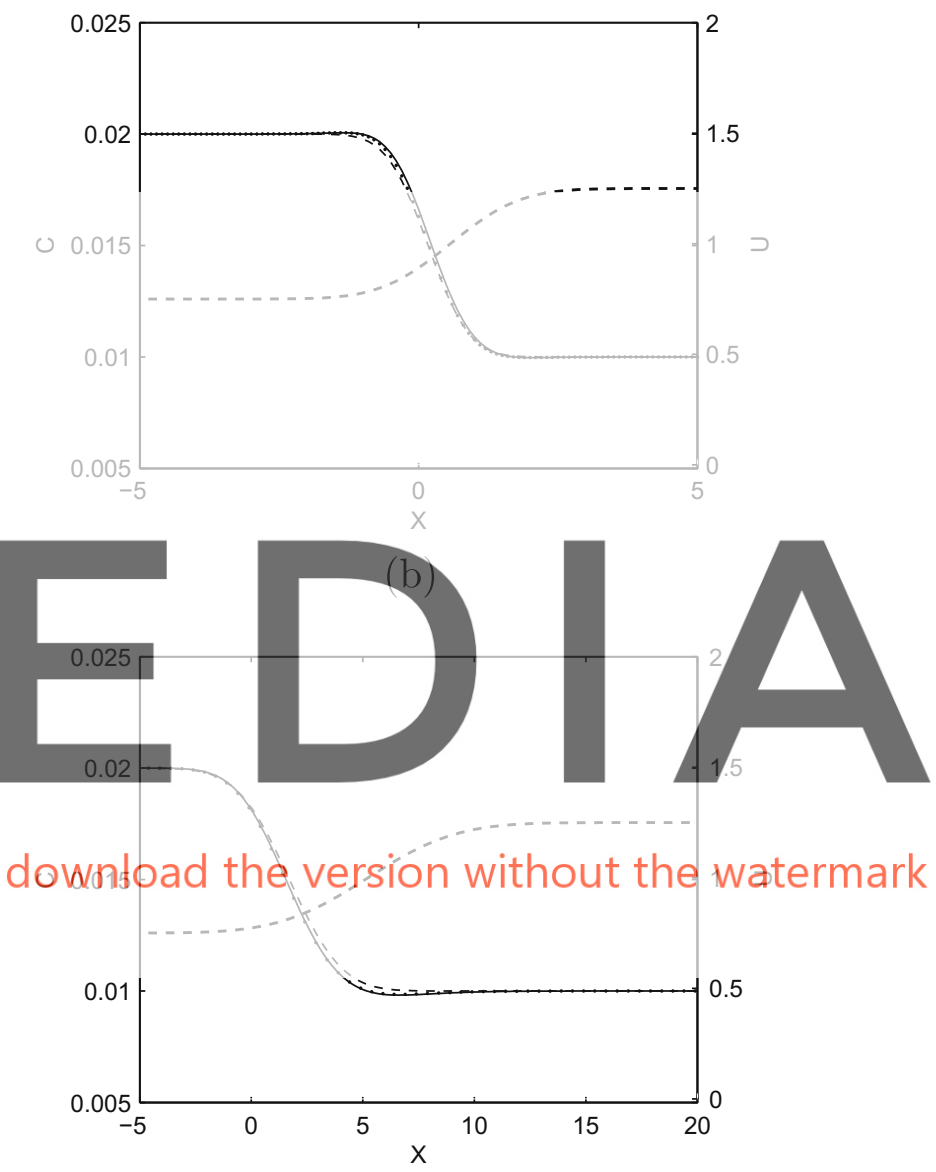

(d)

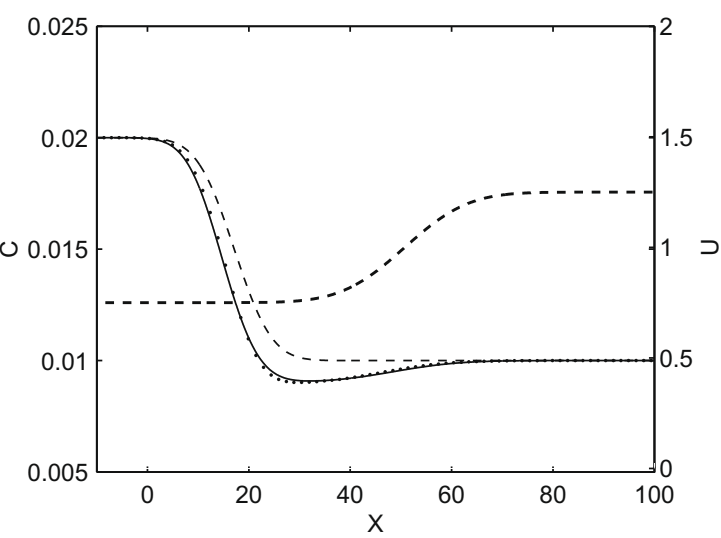

(f)

Fig. 1. Spatial dependence of concentrations $C_{1}$ and $U$ for $U^{\prime}=0.25$ for the case in Table 1 at a series of fixed times. (a) $T=0.1$, (b) $T=0.5$, (c) $T=1$, (d) $T=5$, (e) $T=10$, and (f) $T=50$. (-) Full numerical solution for $C_{1}$. (.) Perturbative solution (up to first order) for $C_{1}(--)$ zeroth order solution for $C_{1}$ and (-- for $U$ ). 
$\frac{\partial \beta_{1}}{\partial c_{1}}=\frac{K u}{\left(u-c_{1}+K c_{1}\right)^{2}}$,

$\frac{\partial \beta_{1}}{\partial u}=\frac{-K c_{1}}{\left(u-c_{1}+K c_{1}\right)^{2}}$.

Introducing (9a) into (1), we can write the governing PDE for $c_{1}$ as

$\left(1+\frac{\gamma}{z_{1}} \frac{K u}{\left(u-c_{1}+K c_{1}\right)^{2}}\right) \frac{\partial c_{1}}{\partial t}-\frac{\gamma}{z_{1}} \frac{K c_{1}}{\left(u-c_{1}+K c_{1}\right)^{2}} \frac{\partial u}{\partial t}$

$=-\mathbf{v} \nabla c_{1}+\nabla\left(\mathbf{D} \nabla c_{1}\right)$.

This is a highly non-linear equation, which is difficult to solve analytically. In the particular case when the absolute value of $c_{1}(K-1)$ is small compared to $u$ we can perform the following approximation $\left(u-c_{1}+K c_{1}\right)^{2} \simeq u^{2}$. This approximation occurs either when $c_{1} \ll c_{2} \sim u$, which depends on the specific problem being considered or if $K \approx 1$, which can occur for example in the Lithium-Sodium case (Appelo and Postma, 2005). Under this approximation (10) becomes

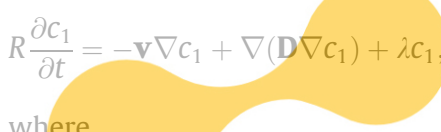

where

$R=1+\frac{\gamma}{z} \frac{K}{u}$

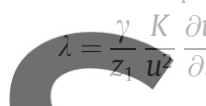

ear in $c_{1}$, while $R(x$

Now (11) is linear in $c_{1}$, while $R(x$, mally incorporates that $\gamma$ may vary

explore the impact upon transport thus a spatially variable re
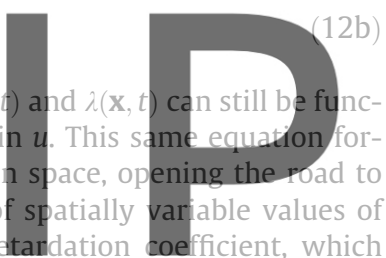

has been shown to play a significant role in breakthrough curve tailing (Dentz and Castro, 2009) and uncertainty (Dentz et al.

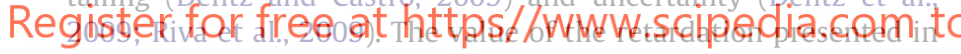

(12a) is slightly different from other expressions available in the literature (e.g. Valocchi et al., 1981; Samper-Calvete and Yang, 2007).

Thus (11) can be read as an advection-dispersion equation with variable retardation plus a (variable) degradation term. The latter term accounts for a reduction of $c_{1}$ whenever $\frac{\partial u}{\partial t}$ is negative, while a positive $\lambda$ value implies a source term. Notice that if $u$ were constant, the degradation term would not be included; this is the case considered in most existing analytical solutions (e.g. Kool et al., 1989; Dou and Jin, 1996; Jin and Ye, 1999).
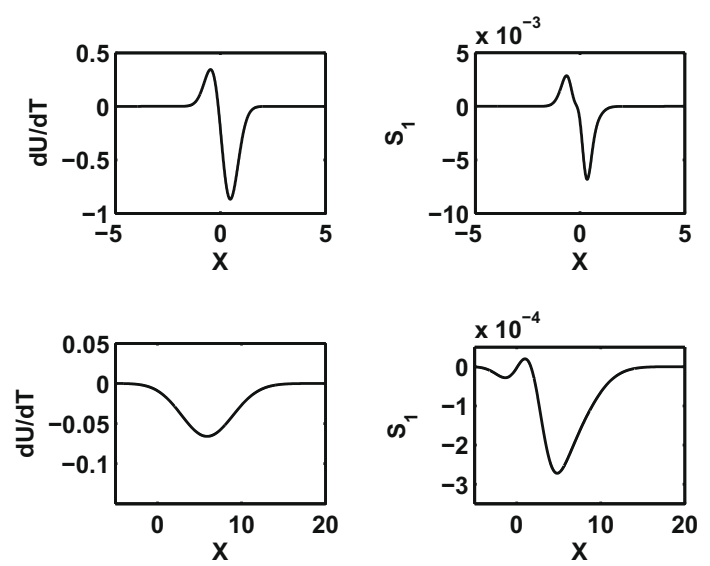

Fig. 2. Plots of $\frac{d U}{d T}$ and $S_{1}$ at two times for the $U^{\prime}=0.25$ case. The top row is for $T=0.1$ and the bottom row for $T=5$.

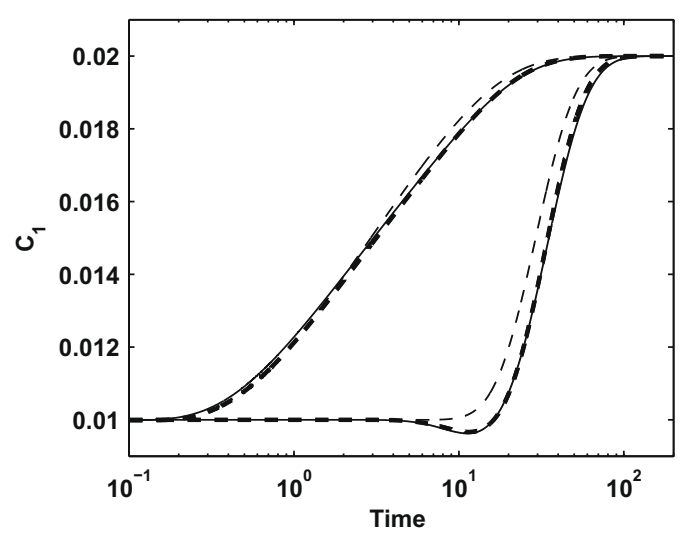

Fig. 3. Breakthrough curves for case in Table 1 for $U^{\prime}=0.25$ at two distances $X=1$ (curves on the left) and $X=10$ (right). (-) Numerical solution, (- -) zeroth order solution and (- -) perturbation solution.

The solution of the full problem consists of the following six steps:

- Solve (7) by means of an analytical solution for some particular case (simple geometry and boundary conditions), or numerically in general; any available code for conservative transport could be used in this step.

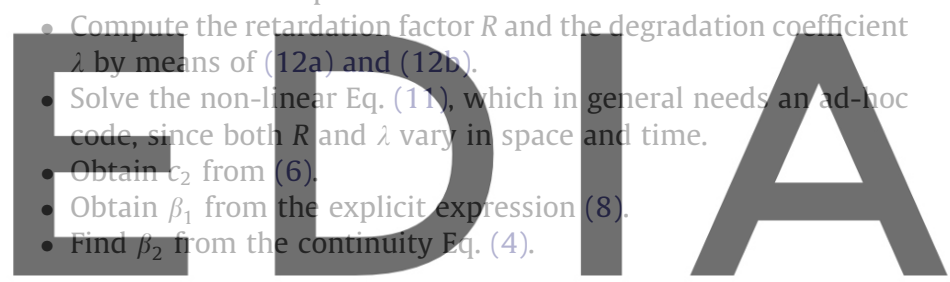

Dimensionless approach

\section{download the version without the watermark}

that $\mathbf{v}=(v, 0,0)$, and $D_{11}=D_{L} ; D_{22}=D_{33}=D_{T}$. Using the following dimensionless variables

$U=\frac{u}{\bar{u}} \quad C_{1}=\frac{c_{1}}{\bar{u}} \quad X=\frac{v}{D_{L}} x \quad Y=\frac{v}{D_{L}} y$

$Z=\frac{v}{D_{L}} z \quad T=\frac{v^{2}}{D_{L}} t$

and the dimensionless quantities $D^{\prime}=D_{T} / D_{L}$, the governing equations can be written in dimensionless form as

$\frac{\partial U}{\partial T}=-\frac{\partial U}{\partial X}+\frac{\partial^{2} U}{\partial X^{2}}+D^{\prime} \frac{\partial^{2} U}{\partial Y^{2}}+D^{\prime} \frac{\partial^{2} U}{\partial Z^{2}}$,

$R \frac{\partial C_{1}}{\partial T}=-\frac{\partial C_{1}}{\partial X}+\frac{\partial^{2} C_{1}}{\partial X^{2}}+D^{\prime} \frac{\partial^{2} C_{1}}{\partial Y^{2}}+D^{\prime} \frac{\partial^{2} C_{1}}{\partial Z^{2}}+\lambda C_{1}$,

where

$R=1+\frac{\gamma}{z_{1}} \frac{K}{\bar{u}} \frac{1}{U}$

$\lambda=\frac{\gamma}{z_{1}} \frac{K}{\bar{u}} \frac{1}{U^{2}} \frac{\partial U}{\partial T}$

and $\bar{u}$ is a characteristic concentration (e.g., some mean concentration).

\section{A perturbative solution to the binary homovalent problem}

As stated before, most existing solutions are based on assuming that $U$ does not change in time. In our formulation this is 
equivalent to assuming that $\lambda=0$. As our model allows for nonzero $\lambda$, if variations in $U$ are small, we can adopt a perturbative solution approach. We start by writing the solution in terms of an expansion

$C_{1}=\sum_{n=0}^{\infty} C_{1, n}$

Assuming that $\lambda$ and variations in $R$ are small, in the 1D case, the governing equation for $C_{1,0}$ is

$\bar{R} \frac{\partial C_{1,0}}{\partial T}+\frac{\partial C_{1,0}}{\partial X}-\frac{\partial^{2} C_{1,0}}{\partial X^{2}}=0$

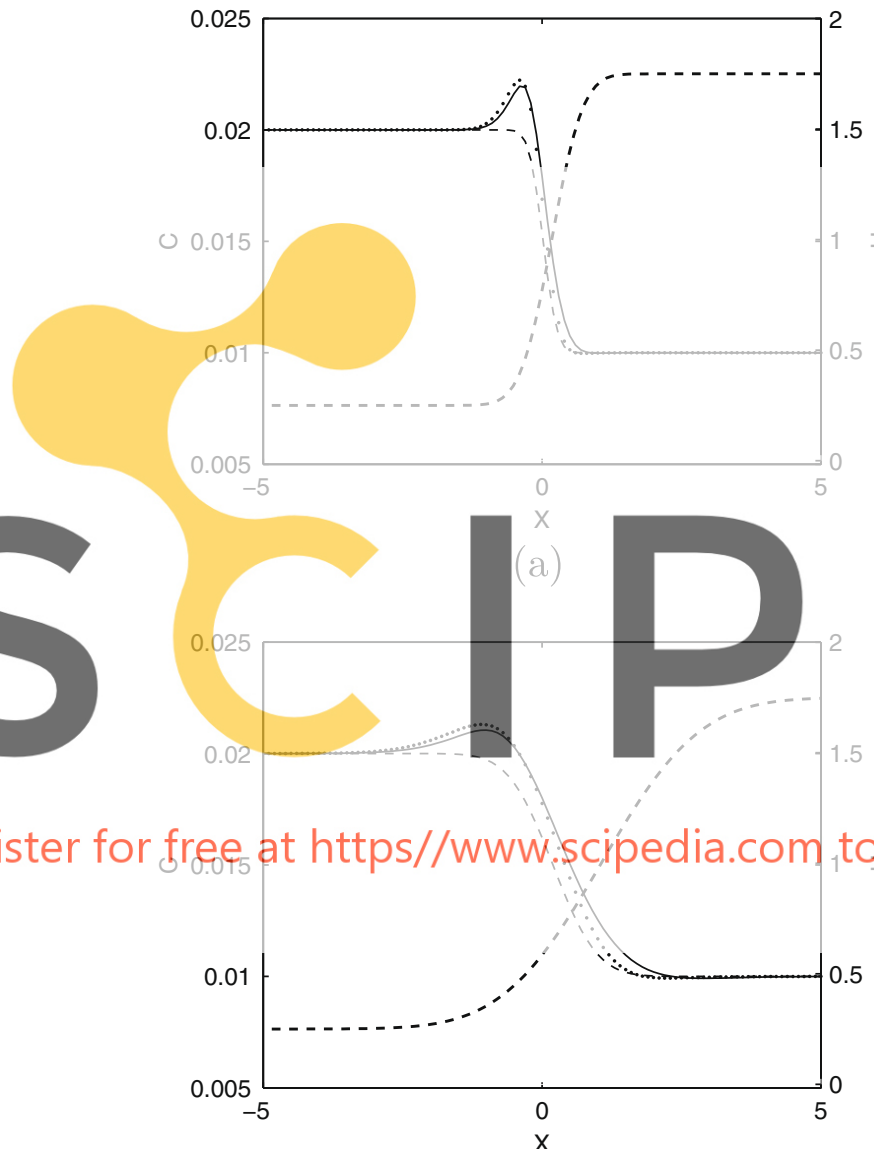

(c)

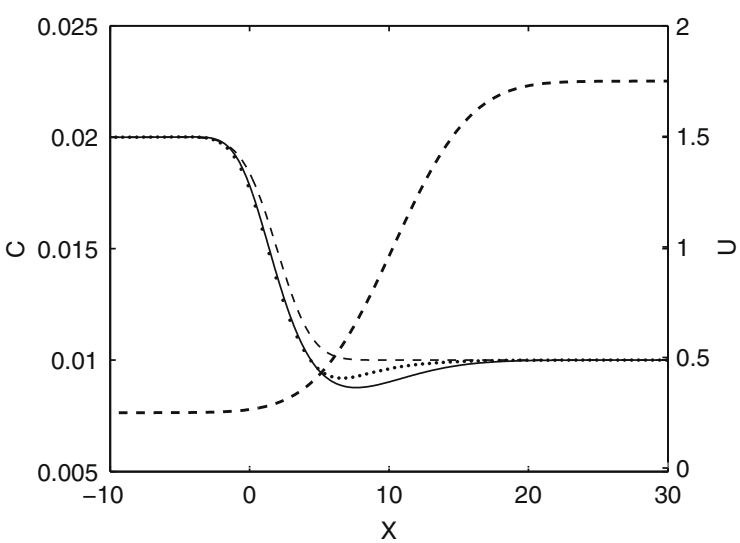

(e) where

$\bar{R}=1+\frac{K \gamma}{z_{1}} \overline{u^{-1}}$

is a mean value of the spatially variable $R$, which in this problem here only varies due to changes in $u$. At first order

$\frac{\partial C_{1,1}}{\partial T}+\frac{1}{\bar{R}} \frac{\partial C_{1,1}}{\partial X}-\frac{1}{\bar{R}} \frac{\partial^{2} C_{1,1}}{\partial X^{2}}=S_{1}$

where
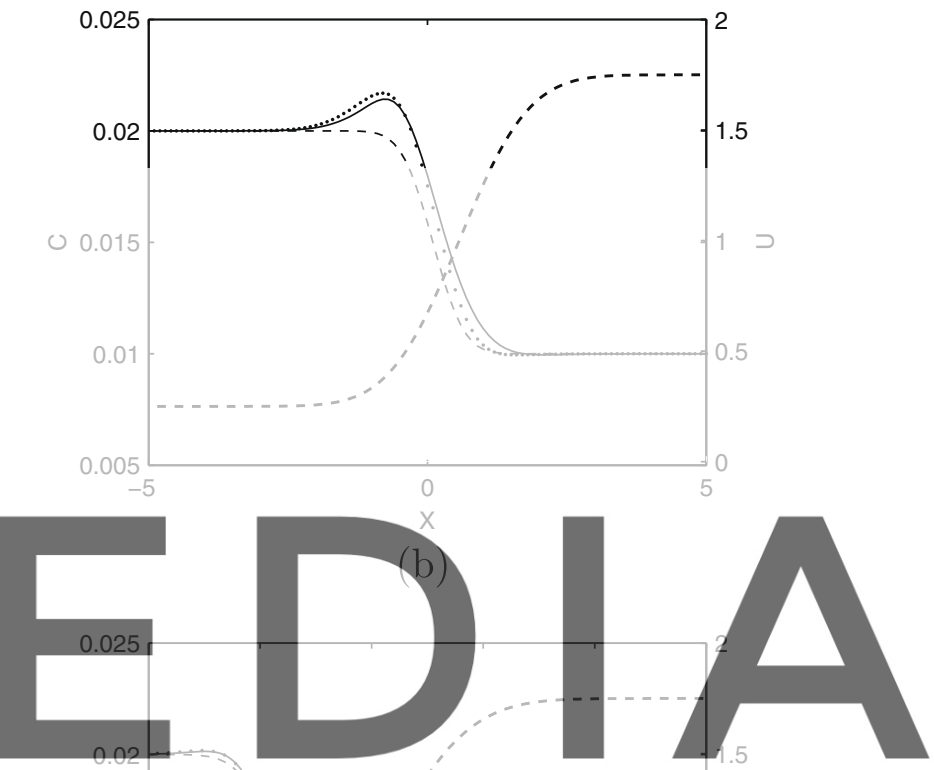

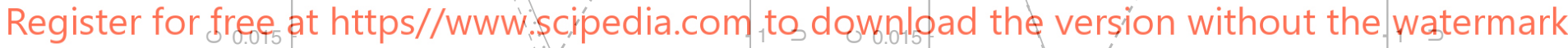

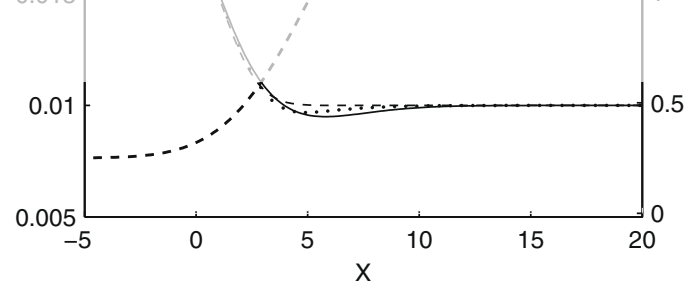

(d)

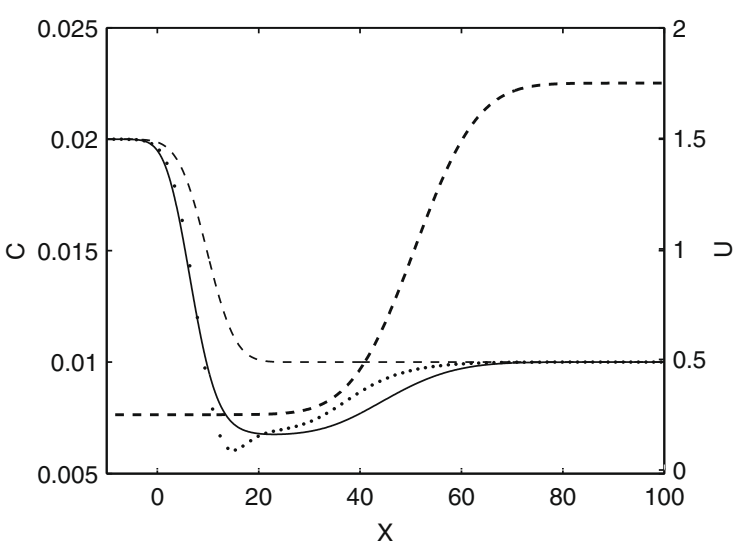

(f)

Fig. 4. Spatial dependence of concentrations $C_{1}$ and $U$ for $U^{\prime}=0.75$ for the case in Table 1 at a series of fixed times. (a) $T=0.1$, (b) $T=0.5$, (c) $T=1$, (d) $T=5$, (e) $T=10$, and (f) $T=50$. (-) Full numerical solution for $C_{1}$. (.) Perturbative solution for $C_{1}$. (--) Zeroth order solution for $C_{1}$ and (- for $U$ ). 
$S_{1}=\frac{\lambda C_{1,0}}{\bar{R}}-\frac{(R-R)}{\bar{R}} \frac{\partial C_{1,0}}{\partial T}$.

Therefore

$C_{1,1}=\int_{0}^{T} \int_{-\infty}^{\infty} S_{1}(\xi, \tau) G(X, \xi, T-\tau) d \xi d \tau$

where $G$ is the Greens function associated with the advection-diffusion-retardation equation, given by (Leij et al., 2000).

$G(X, \xi, \tau)=\sqrt{\frac{\bar{R}}{4 \pi \tau}} \exp \left(\frac{-\bar{R}\left(X-\xi-\frac{1}{\bar{R}} \tau\right)^{2}}{4 \tau}\right)$

While we restrict our analyses to first order corrections, if deemed necessary, higher order corrections could easily be implemented and are given by

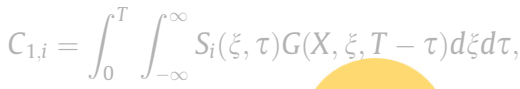

where

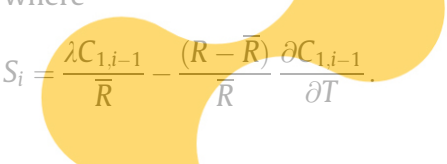

Illustrative example

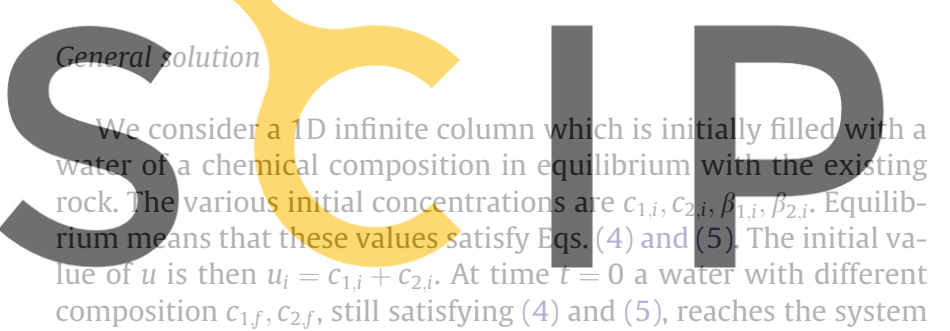

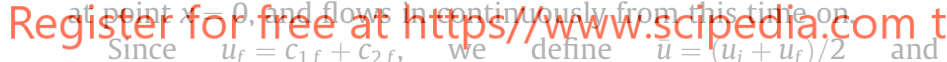

$u^{\prime}=\left(u_{i}-u_{f}\right) / 2$ (and $\left.U^{\prime}=u^{\prime} / \bar{u}\right)$. Working in dimensionless variables, the solution for $U$ is

$U=1+U^{\prime} \operatorname{erf}\left(\frac{X-T}{\sqrt{4 T}}\right)$.

From (18), the solution for $C_{1,0}$ is

$C_{1,0}=\frac{C_{1 i}+C_{1 f}}{2}+\frac{C_{1 i}-C_{1 f}}{2} \operatorname{erf}\left(\frac{X-a T}{\sqrt{4 a T}}\right)$
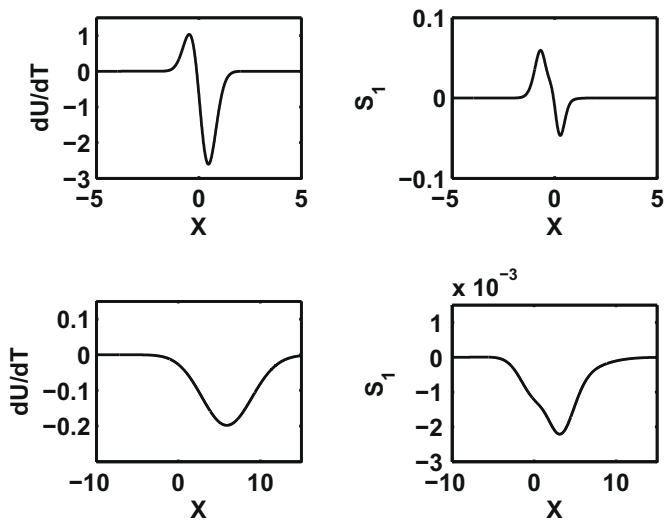

Fig. 5. Plots of $\frac{d U}{d T}$ and $S_{1}$ at two times for the $U^{\prime}=0.75$ case. The top row is for $T=0.1$ and the bottom row for $T=5$. where $a=\bar{R}^{-1}$. The solution for $C_{1,1}$ is sought by integration of (22). The quadrature is done numerically using standard numerical integration methods.

Here, we present two cases for the conditions considered in Table 1 . The first is for $U^{\prime}=0.25$ and the second for $U^{\prime}=0.75$. As $U^{\prime}$ becomes larger (approaches 1 which is its maximum possible physical value) one expects the perturbation approach to become less and less valid. Note that the selected values of $C_{1 i}$ and $C_{1 f}$ are small so as to satisfy our assumption that $c_{1} \ll u$. The influence of this assumption will be presented in more detail further on.

For comparison purposes we also solve the full problem numerically - i.e. we solve (10), which is free of any simplifying assumptions. This is done with an in house finite difference code. The numerical scheme uses central differences in space and forward differences in time (e.g. Pozrikidis, 1998).

Fig. 1 shows the concentration distributions for $C_{1}$, calculated numerically and analytically at various dimensionless times. The analytical solutions presented are for the zeroth order perturbation from Eq. (27), which neglects the influence of $\lambda$ and spatial variability in the retardation coefficient, as well as the solution that includes a first order correction from (22). The plots also show the temporal evolution of the spatial distribution of $U$ in order to explicitly illustrate both the retardation and the temporal change of the source term $\lambda$, which depends both on $U$ and on $\frac{d U}{d T}$.

For the early time snapshots in Fig. 1 it is difficult to distinguish between the three concentration curves, indicating that the zeroth order term is capable of approximately capturing the shape of the

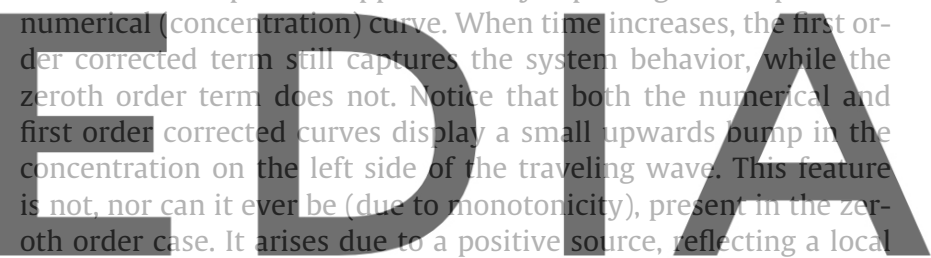
positive value of $\frac{d U}{d T}$. In order to illustrate this a plot of $\frac{d U}{d T}$ and the source term $S_{1}$ (provided by (21)) at two times are shown in Fig. 2.

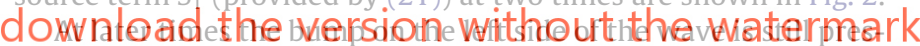
ent, but its value is very close to zero, so in practical terms it disappears. Equivalently, a downwards bump is always present at the front part of the wave. This undershoot is given by two complimentary superposing effects, corresponding to the two source terms in (22). The first effect reflects negative values of $\frac{d U}{d T}$, which leads to negative values of $\lambda$. Note that at these late times $\frac{d U}{d T}$ is either negative or very close to zero. This is illustrated nicely in the late time plot in Fig. 2. The influence of this source tends to be at the front edges of influence as the wave associated with $U$ travels fastest at the full flow speed. A close inspection of Fig. 1

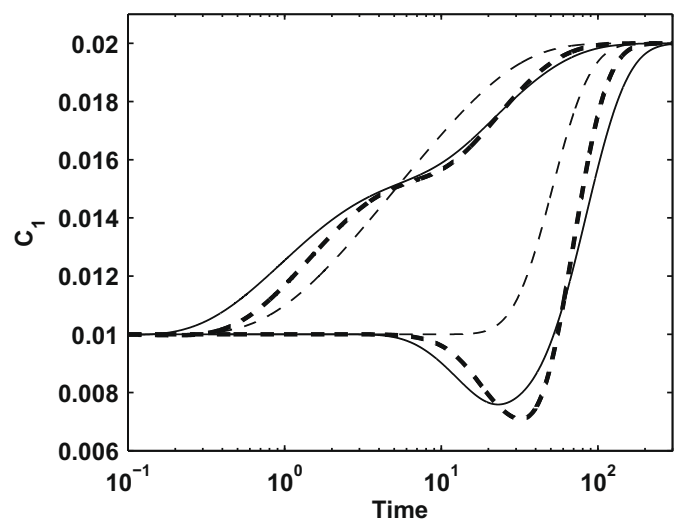

Fig. 6. Breakthrough curves for the example corresponding to $U^{\prime}=0.75$ at two distances $X=1$ and $X=10$. (-) Numerical solution, (- $)$ zeroth order solution and (- -) perturbation solution. 
reveals that the leading edge of the downward bump coincides with the leading edge of the $U$ wave.

The second effect stems from the fact that spatial variations in $U$ lead to spatial changes in the retardation coefficient with greater retardation on the left hand side of the $U$ wave (corresponding to smaller values of $U$ ). In a similar manner that the first contribution from (22) depends on temporal variations in $U$, the second terms depends on temporal changes in $C_{1}$. As $C_{1}$ is traveling at a retarded speed relative to $U$ its region of influence is on the rearward side and leads to negative contributions here. This is reflected by the fact that the numerical and perturbation solution appear to be traveling slower than the zeroth order case and that the trough of the downwards bump is to the left of the zeroth order solution. These features are particularly evident at later times. Once again, due to the monotonic nature of the zeroth order solution for concentration such features can never be captured. However, with our proposed first order correction, the full numerical solution seems to be well captured both qualitatively and quantitatively.

In order to further illustrate these features breakthrough curves of $C_{1}$ for the three solutions at two points $(X=1$ and $X=10)$ are shown in Fig. 3. For the early breakthrough curve at $X=1$ the differences between the three cases are subtle, reflecting the small differences observed at early times in Fig. 1. None the less there are differences and the curve corresponding to the first order

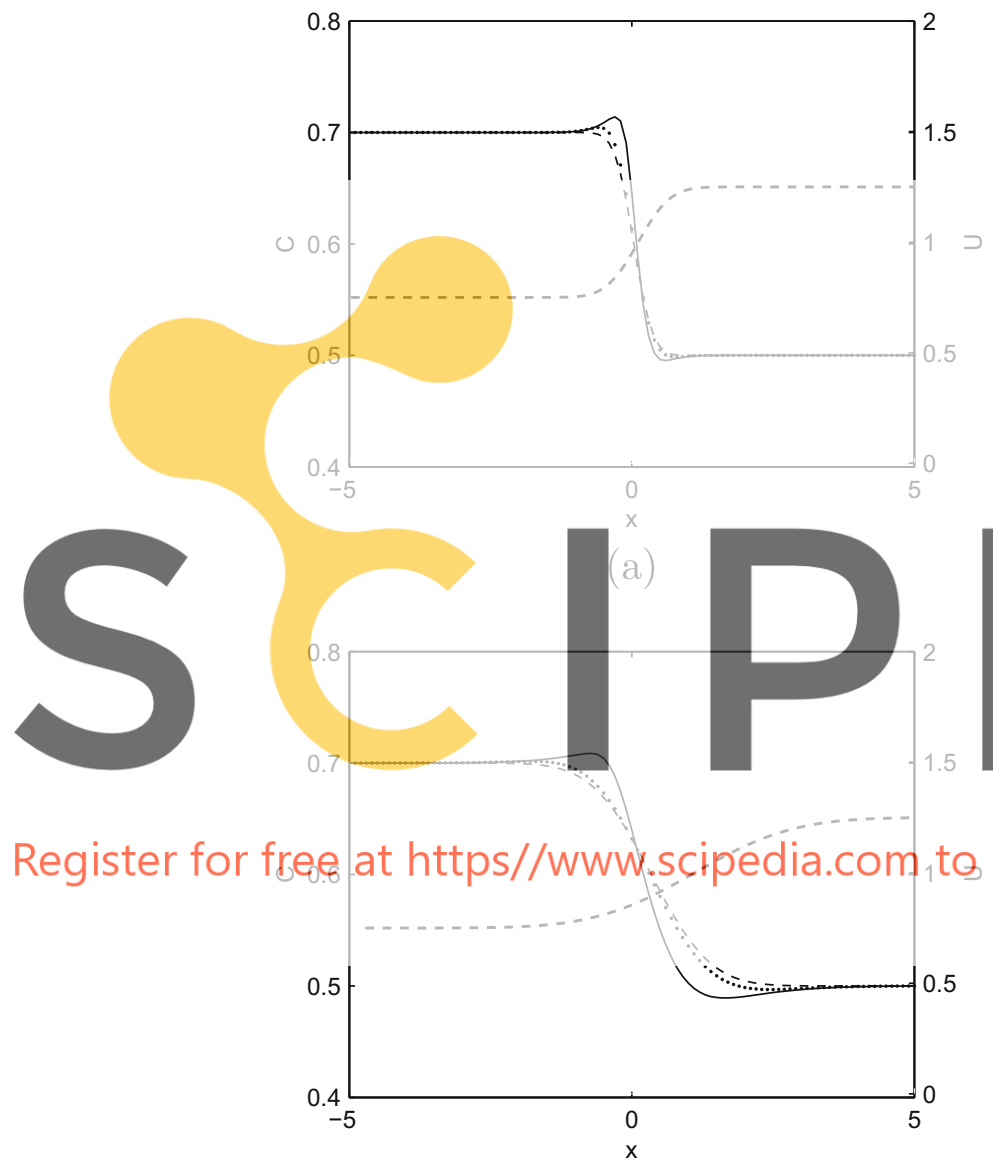

(c)

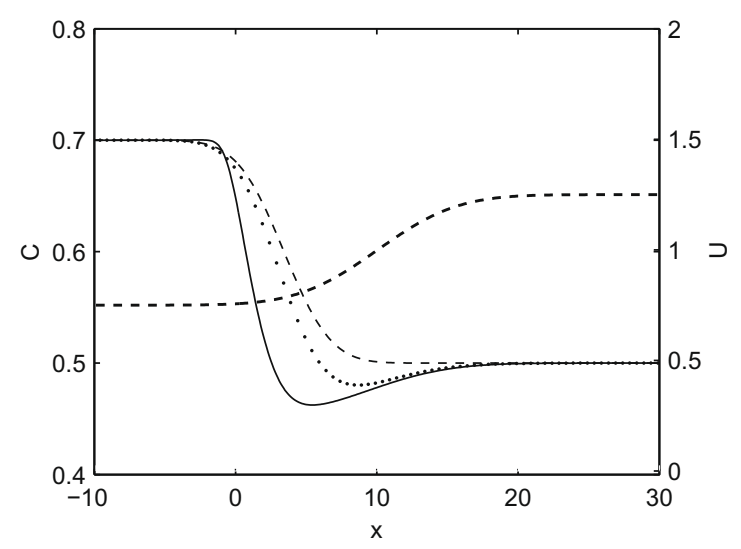

(e)

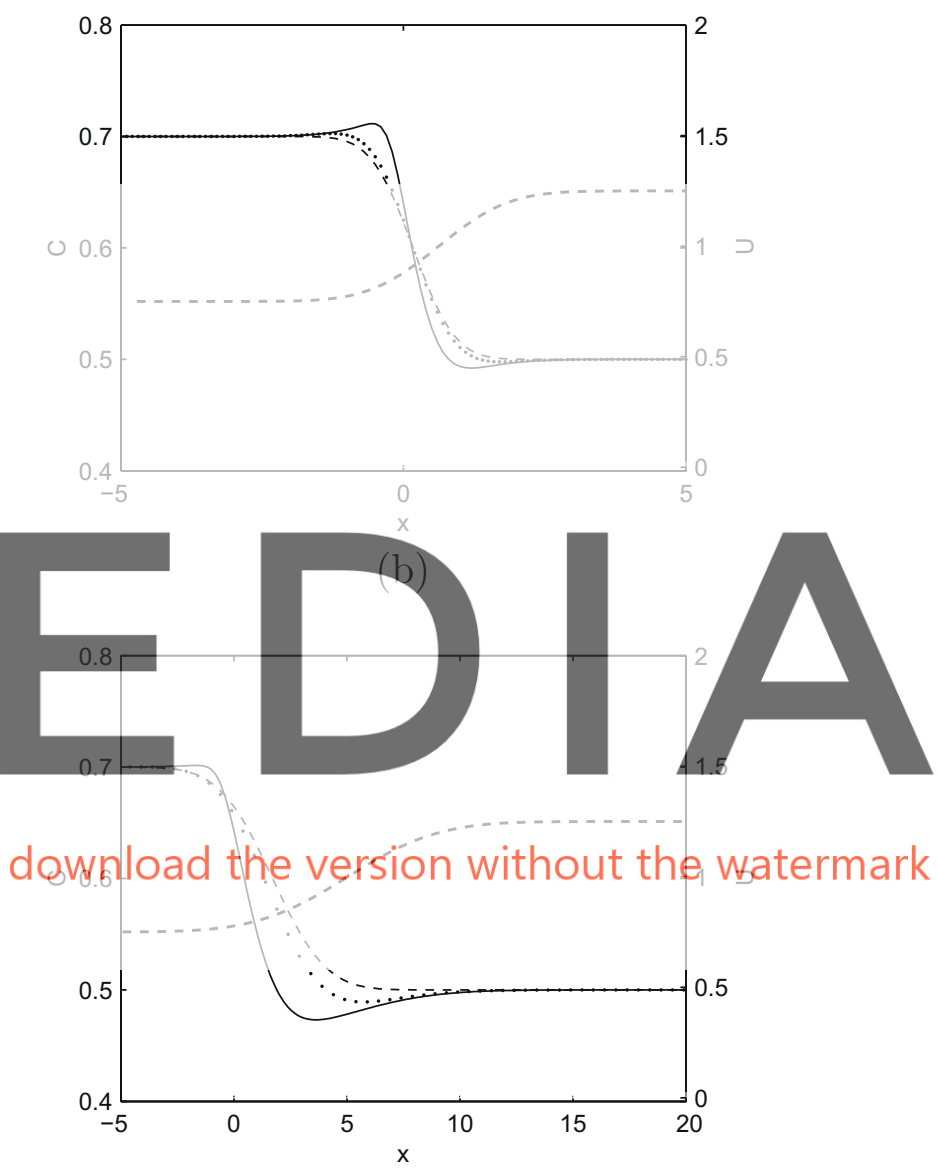

(d)

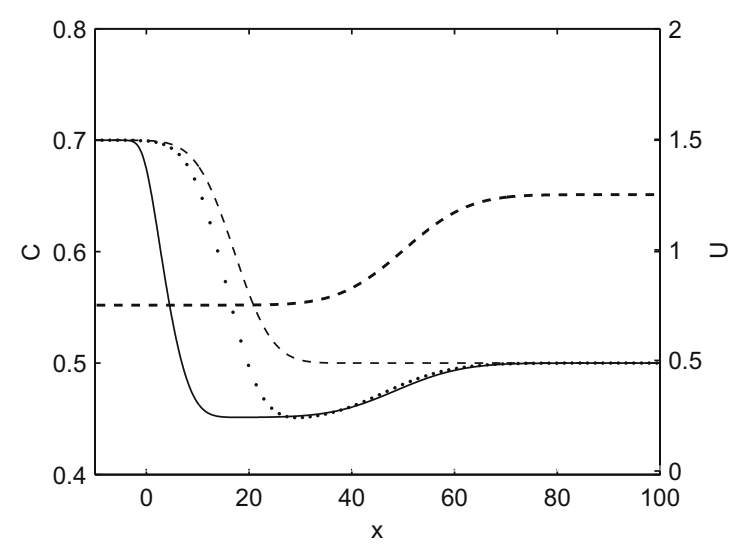

(f)

Fig. 7. Spatial dependence of concentrations $C_{1}$ and $U$ for $U^{\prime}=0.25$ for the modified case (large $C_{1}$ values) at a series of fixed times: (a) $T=0.1$, (b) $T=0.5$, (c) $T=1$, (d) $T=5$, (e) $T=10$, and (f) $T=50$. (-) Full numerical solution for $C_{1}$. (.) Perturbative solution for $C_{1}(--)$ zeroth order solution for $C_{1}$ and $(--$ for $U)$. 
solution agrees more favourably with the full numerical case than the zeroth order solution. The differences between the solutions is far more evident for the later breakthrough curve at $X=10$. This is particularly evident for the decrease in concentration at a dimensionless time close to one predicted in the numerical and corrected concentration values.

A similar set of concentration profiles and breakthrough curves for the $U^{\prime}=0.75$ case are shown in Figs. 4-6. This time, due to the larger value of $U^{\prime}$ the upwards bump on the left hand side of the traveling wave is far more evident, which the zeroth order analytical solution can never reproduce. However, despite the quite large value of $U^{\prime}$ (physically it must be less than 1 so a value of 0.75 can be regarded as relatively large), the first order correction does a reasonable job, both qualitatively and quantitatively of capturing this effect. Once again, at later times the undershoot on the right hand side of the wave appears. This time the corrected analytical solution can only qualitatively reproduce this feature. This is unsurprising as perturbative approaches to advection-diffusion equations are known to suffer from problems when the perturbations are no longer smahl (e.g. Jarman and Tartakovsky, 2008). It is possible to go to higher orders in the perturbation and out of academic interest we did so. We found that the undershoot is indeed well captured by going to higher order corrections (third or fourth order is required). However, as this requires the numerical quadrature of already numerically calculated corrections it raises the question of its utility as most of the fundamental physical processes can be captured qualitatively with only a correction at first

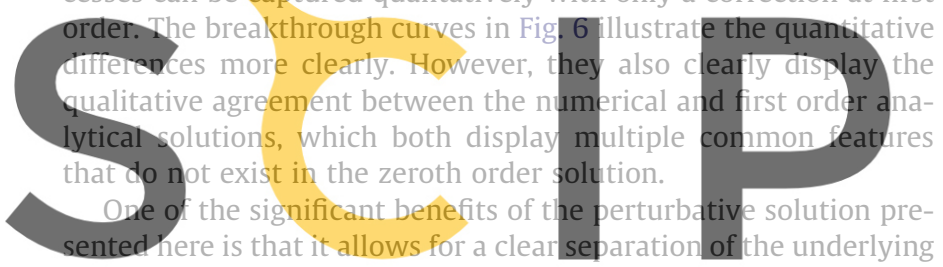
physical processes. This allows us to identify which specific process leads to the specific structures observed in the solution curves

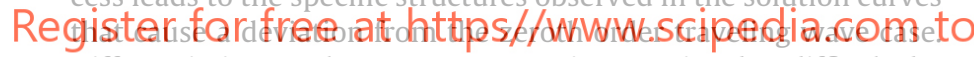
Differentiating such processes can in practice be difficult by numerical procedures only. Additionally, such solution approaches can provide essential tools in the field of probabilistic risk assessment (e.g. Tartakovsky, 2007; Bolster et al., 2009), where simplified models are systematically linked together as building blocks to study coupled larger scale problems and asses risks.

\section{Influence of small $c_{1}$ approximation}

In the previous section we illustrated that our analytical solution does a reasonable job of reproducing a full numerical solution of the problem presented. However in going from (10) to (11) we made a strong approximation in assuming that $u \gg|1-K| c_{1}$ and here we illustrate by an example what occurs when this approximation is not valid. The case considered is identical to that described in Table 1 with the exception that now $C_{1 i}=0.7$ and $C_{1 f}=0.5$, clearly violating our approximation. The $U^{\prime}=0.25$ case, where our analytical solution worked well before, is considered. Concentration profiles at various times are shown in Fig. 7. While many of the features that were present before (bumps on the front and back of the waves) are still present both in the full solution and our corrected analytical solution, any agreement can really only be said to be qualitative. In particular it is very evident that our model does not capture the enhanced retardation of the traveling wave (best seen in Fig. 7f). While the retardation is better than for the case that completely neglects spatial variations in $U$ it simply does not diminish sufficiently as the influence of $C_{1}$ on it (which we neglect) clearly plays an important role. This motivates the need to find solutions where no such approximation is necessary. How- ever, at this point, none are currently obvious to us and thus we can only present further food for thought. Note that in this particular case, had we chosen $K \approx 1$, our linearisation approximation would have been valid, leading to good agreement between the perturbation and numerical solutions (as was seen in Fig. 1).

\section{Conclusions}

We present a formulation for decoupling the transport equations that govern homovalent cation exchange. Contrary to previously existing solutions, our methodology allows for the presence of an injected water whose total concentration of cations (or charges) of the aqueous species varies over time (i.e. in our notation $u$ does not have to be constant). The inclusion of such a variation introduces further non-linearity into the governing equations for the individual species, but most notably, incorporates a degradation/production term in the equation which is written in terms of the time derivative of the total cation concentration of the input water.

The solution to the problem is sought in a step-by-step fully decoupled methodology. There is still a major drawback as one needs to solve a highly non-linear equation for the aqueous species concentration. In order to circumvent this we propose an approximation that is valid when the concentrations of one of the species is much larger than the other or when $K$, the Gaines-Thomas equilibrium constant, is close to unity. The resulting equation is a linear advection-teaction-diffusion equation with spatio-tempofally var-
iable retardation and degradation coefficients. If changes in the to-
tal concentration are not large, then the system can be folyed with
a perturbative approach, which assumes a retarded trave/ing wave
solution at zeroth order that is perturbed by changes in the retar-
dation coefficient as well as the temporally varying degradation
term, which is diven by the non retarder total concentration wave.

The physical mechanisms of what occurs can be understood by

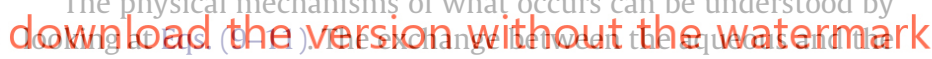
adsorbed phase has two principle effects. The first is on the retardation of the cation concentration such as is quantified by the retardation coefficient $R$. The second influence appears as a transient "reactive" term quantified by $\lambda$. The larger the total concentration of cations in the system is (i.e. $U$ ) the less the retardation $R$. Intuitively this makes sense as less cation concentration in the water leads in proportional terms to more cations being immobilized (since we consider the full CEC to be used). In other terms, retardation defined as the fraction of time the solute stays immobilized with respect to the time in which the solute is mobile, increases, causing a decrease in effective velocity. Thus, when water with a different $U$ is introduced into the system as is the case in the examples here there are two distinct travel speeds. In these particular case $U$ is decreased, which means that the effective velocity entering is less than that which was initially there. Consequently the two traveling waves tend to separate more and more, causing a physical "hole"/dip in the distribution of $C_{1}$ in space. Physically, the fact that this effective velocity varies in space means that it is not incompressible and that therefore regions of accumulation and decumulation can occur such as observed by the bumps and valleys depicted here.

The $\lambda$ term reflects regions of non-uniform total concentration $U$ and accounts for the transient adjustment of the system from one state to another. The terms can be positive or negative depending whether the total concentration is increasing or decreasing. Physically this term is equivalent a having a sucking out or injection of concentration from the adsorbed phase to the aqueous phase. In our particular case water with a lower $U$ value enters the system. This is equivalent a having a term sucking out concentration 
at the front of the wave and injecting in behind it. This mechanism can be self compensating depending on the choice of boundary conditions.

In order to illustrate the methodology we have presented a onedimensional example of an infinite column that is then invaded by a water of different chemical composition. We also solve the problem numerically in order to compare results. For the case where the existing and the injected water have similar (but not equal) total cation concentration, the perturbation approach produced excellent qualitative and quantitative agreement with the full numerical simulation, particularly compared with the zeroth order traveling wave solution at late times. In particular the perturbation approach is able to faithfully capture certain features (bumps and valleys), which are not possible in the monotonic traveling wave case. For larger variations of the total concentration, where one might expect the perturbative approach to no longer be valid, the perturbation approach still outperforms the traveling wave case significantly, qualitatively reproducing many of the features in the numerical solution. Unfortunately, due the invalidity of the perturbation assumption, the quantitative agreement at late times is not great. At early times it still seems to work very well.

Finally, in order to illustrate the influence of our linearisation of the problem we present a case where the assumption leading to the linearisation is clearly not valid. In this case our solution approach can not accurately reproduce the real retardation in the system resulting in a front that travels at the wrong speed. The result is still better than the zeroth order traveling wave case, but quan-
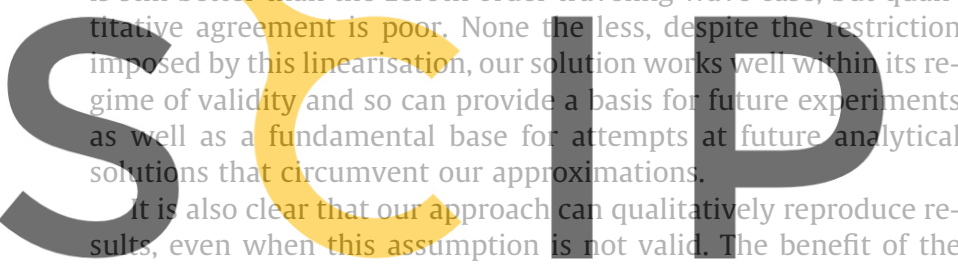
perturbation approach is that it allows for a clear separation of the underlying physical processes which can allow one to identify

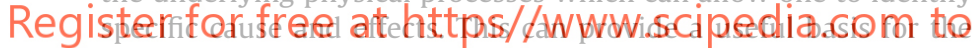
interpretation of experimental and field scale results, the assessment of risk and also provides a solid foundation for the design of future experiments.

\section{Acknowledgements}

Financial support from the EU Project Gabardine is gratefully acknowledged. DB would like to thank the Spanish ministry of science for funding him and this project via the Juan de la Cierva program.

\section{References}

Appelo, C., 1994a. Cation and proton exchange, $\mathrm{pH}$ variations, and carbonate reactions in a freshening aquifer. Water Resources Research 30, 2793-2805.

Appelo, C., 1994b. Some calculations on multicomponent transport with cationexchange in aquifers. Ground Water 32 (6), 968-975.

Appelo, C., Hendriks, J., Vanveldhuizen, M., 1993. Flushing factors and a sharp front solution for solute transport with multicomponent ion-exchange. Journal of Hydrology 146, 89-113.

Appelo, C., Postma, D., 2005. Geochemistry,Groundwater and Pollution. CRC Press.

Bhattacharyya, A., Mohapatra, P., Pathak, P., Manchanda, V., 2006. Cation-exchange separation of uranium from thorium in nitric acid medium. Journal of Radioanalytical and Nuclear Chemistry 268 (2), 323-328.

Bjerg, P., Christensen, T., 1993. A field experiment on cation exchange-affected multicomponent solute transport in a sandy aquifer. Journal of Contaminant Hydrology 12 (4), 262-290.

Bolster, D., Tartakovsky, D., Dentz, M., 2007. Analytical models of contaminant transport in coastal aquifers. Advances in Water Resources 30, 1962-1972.

Bolster, D., Barahona, M., Dentz, M., Fernandez-Garcia, D., Sanchez-Vila, X., Trinchero, P., Valhondo, C., Tartakovsky, D.M., 2009. Probabilistic risk analysis of groundwater remediation strategies. Water Resources Research 45, W06413. doi:10.1029/2008WR007551.
Bouwer, H., 2002. Artificial recharge of groundwater: hydrogeology and engineering. Hydrogeology Journal 10, 121-142.

Carlyle, H., Tellam, J., Parker, K., 2004. The use of laboratory-determined ion exchange parameters in the predictive modelling of field-scale major cation migration in groundwater over a 40-year period. Journal of Contaminant Hydrology 68, 58-81.

Cernik, M., Barmettler, K., Grolimund, D., Rohr, W., Borkovec, M., Sticher, H., 1994. Cation-transport in natural porous-media on laboratory-scale multicomponent effects. Journal of Contaminant Hydrology 16, 319-337.

Dentz, M., Bolster, D., le Borgne, T., 2009. Concentration statistics for transport in a random medium. Physical Review E 80, 010101.

Dentz, M., Castro, A., 2009. Effective transport dynamics in porous media with heterogeneous retardation properties. Geophysical Research Letters 36, L03403.

Dou, W., Jin, Y., 1996. Analytical solution of the solute transport equation for the binary homovalent ion exchange in groundwater. Journal of Hydrology 180, 139-153.

Gaines, G., Thomas, H., 1953. Adsorption studies on clay minerals, ii, a formulation of the thermodynamics of exchange adsorption. Journal of Chemical Physics 21 , 714-718.

Harmsen, K., Bolt, G., 1982. Movement of ions in soild, i. ion exchange and precipitation. Geoderma 28, 85-101.

Jarman, K.D., Tartakovsky, A.M., 2008. Divergence of Solutions to Solute Transport Moment Equations. Geophysical Research Letters 35, 30-34. L15401.

Jin, Y., Ye, S., 1999. Analytical solution for monovalent divalent ion exchange transport in groundwater. Canadian Geotechnology Journal 36, 1197-1201.

Kool, J., Parker, J., Zelazny, L., 1989. On the estimation of cation exchange parameters from column displacement experiments. Soil Science Society of America Journal 53, 1347-1355

Lambrakis, N., 2006. Multicomponent heterovalent chromatography in aquifers. Modelling salinization and freshening phenomena in field conditions. Journal of Hydrology 323, 230-243.

Leij, F., Priesack, E., Schaap, M., 2000. Solute transport modeled with Greens functions with application to persistent solute sources. Journal of Contaminant Hydrology 41, 155-173.

Lewis, F., Voss, C., Rubin, J., 1987. Solute transport with equilibrium aqueous

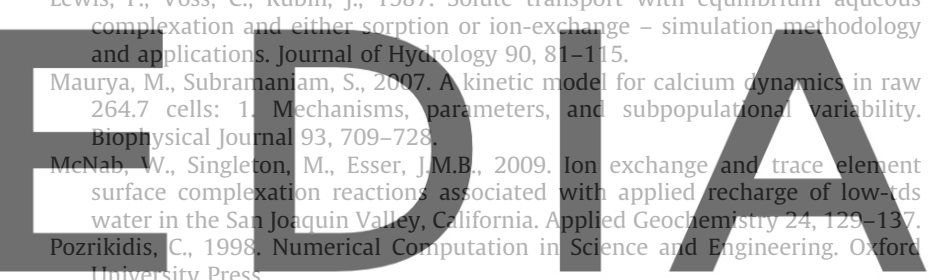

Riva, M., Guadagnini, A., Sanchez-Vila, X., 2009. Effect of sorption heterogeneity on moments of solute residence time in convergent flows. Mathematical

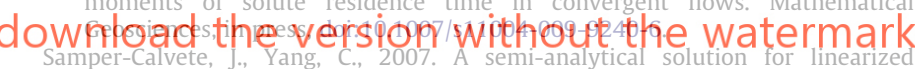
multicomponent cation exchange
Transport in Porous Media 69, 67-88.

Schuin, R., Papritz, A., Fihier, H., Selim, H., 1989. Caicium and magnesium transport in aggregated soils at variable ionic strength. Geoderma 44, 129-141.

Shephard, J., McQuillan, A., Bremer, P., 2008. Mechanisms of cation exchange by Pseudomonas aeruginosa pao1 and pao1 wbpl, a strain with a truncated lipopolysaccharide. Applied and Environmental Microbiology, 6980-6986.

Steefel, C., Carroll, S., Zhao, P., Roberts, S., 2003. Cesium migration in hanford sediment: a multisite cation exchange model based on laboratory transport experiments. Journal of Contaminant Hydrology 67, 219-246.

Tartakovsky, D.M., 2007. Probabilistic risk analysis in subsurface hydrology. Geophysical Research Letters, L05404.

Timms, W., Hendry, M., 2007. Quantifying the impact of cation exchange on longterm solute transport in a clay-rich aquitard. Journal of Hydrology 332, 110122.

Turmanova, S., Godjevargova, T., Vasileva, N., 2005. Immobilization of urease on cation-exchange membranes prepared by radiation-initiated graft copolymerization of acrylic acid on polythene thin films. Polymer Bulletin 55, 467-475.

Valocchi, A., Street, R., Roberts, P., 1981. Transport of ion-exchanging solutes in groundwater: chromatographic theory and field simulation. Water Resources Research 17, 1517-1527.

Vulava, V., Kretzschmar, R., Barmettler, K., Voegelin, A., Grolimund, D., Borkovec, M., 2002. Cation competition in a natural subsurface material: prediction of transport behavior. Water Resources Research 38. doi:10.1029/2001WR000262.

Vulava, V., Kretzschmar, R., Rusch, U., Grolimund, D., Westall, J., Borkovec, M., 2000. Cation competition in a natural subsurface material: modelling of sorption equilibria. Environmental Science and Technology 34, 2149-2155.

Walraevens, K., Cardenal-Escarcena, J., van Camp, M., 2007. Reaction transport modelling of a freshening aquifer (Tertiary Ledo-Paniselian Aquifer, Flanders, Belgium). Applied Geomchemistry 22, 289-305.

Wu, J., Liu, C., Chu, K., Suen, S., 2008. Removal of cationic dye methyl violet $2 \mathrm{~b}$ from water by cation exchange membranes. Journal of Membrane Science 309, 239245.

Xing, Z., Zhao, H., Wu, J., 2006. Reversible exchange of tl and hg cations on the superconducting 1212 lattice. Advanced Materials 18 (20), 2743-2746. 